Review

\title{
Automatic Methods for Generation of Type-1 and Interval Type-2 Fuzzy Membership Functions
}

\author{
Andréia Alves dos Santos Schwaab, Silvia Modesto Nassar and Paulo José de Freitas Filho \\ Department of Informatics and Statistics, Federal University of Santa Catarina, Florianópolis, Brazil
}

\author{
Article history \\ Received: 24-08-2015 \\ Revised: $12-09-2015$ \\ Accepted: 24-10-2015 \\ Corresponding Author: \\ Andréia Alves dos Santos \\ Schwaab \\ Department of Informatics and \\ Statistics, Federal University of \\ Santa Catarina, Florianopolis, \\ Brazil \\ Email:alves.andreia@yahoo.com.br
}

\begin{abstract}
Generation of membership functions is an important step in construction of fuzzy systems. Since membership functions reflect what is known about the variables involved in a problem, when they are correctly modeled the system will behave in the manner that is expected in the context of the problem being addressed. Since their creation, type-1 membership functions have been used in domains characterized by uncertainty. Nevertheless, use of type- 2 membership functions has been expanding over recent years because they are considered more appropriate for this application. Both types of membership function can be generated with the aid of automatic methods that implement generation of membership functions from data. These methods are convenient for situations in which it is not possible to obtain all the information needed from an expert or when the problem in question is complex. The aim of this study is to present a review of the most important automatic methods for generation of membership functions, both type 1 and interval type-2, highlighting the principal characteristics of each approach.
\end{abstract}

Keywords: Type-1 Membership Function, Interval Type-2 Membership Function, Automatic Methods

\section{Introduction}

Fuzzy set theory was created by Zadeh (1965) and since then it has been applied to modeling of problems characterized imprecision or vagueness. It has contributed to many practical domains, such as, for example, medicine, economics, engineering, etc.

A fuzzy inference system is comprised of variables and a rule base. The variables are represented by one or more fuzzy sets, which particularize knowledge about that domain. According to Ross (2010), the membership functions describe what is known about a fuzzy set.

Definition of membership functions is therefore a very important step in designing fuzzy systems, because it is part of the knowledge base needed to make inferences and, consequently, achieve the desired results. It is through membership functions that knowledge about the variables involved in a problem is specified. The most commonly employed types of membership function are Gaussian, Triangular and Trapezoidal. Definition of functions can be based on the knowledge of experts on the subject in question or can be performed with the aid of automatic methods.
The membership functions most commonly employed in fuzzy systems are those known as type-1 functions. In a type-1 fuzzy membership function, each element of the problem domain has a degree of membership in the fuzzy set. This degree of membership is a real value in the range $[0,1]$. However, this characteristic has been identified as providing an inadequate representation of uncertainty, because it indicates with exactitude the degree of an element's membership of the fuzzy set (Klir and Yuan, 1995; Mendel, 2003). Type-1 fuzzy sets are appropriate when the knowledge available for modeling is adequate to determine elements' membership of the fuzzy set (Ross, 2010).

Sometime after their initial work on fuzzy sets, Zadeh (1975) published a work that takes into account the uncertainty in defining the degree of membership of an element in a fuzzy set. Thus, he introduced type2 fuzzy sets, which are sets whose membership degree of an element is a type-1 fuzzy set. This means that instead of a real value, the degree of membership can be a type-1 fuzzy set. A type-2 fuzzy set is represented by a type-2 fuzzy membership function. 
Type-2 fuzzy sets have greater power to represent uncertainty, since they have more degrees of freedom and are three-dimensional (Mendel and John, 2002). According to Liang and Mendel (2000), the occurrence of uncertainty in modeling fuzzy systems can be identified: (a) in the definition of linguistic concepts, the meanings of which may be different for different people; (b) in the definition of the consequences of rules, which also may not be a common choice within a group of individuals; (c) when there is noise in the data. The use of type- 2 sets to represent uncertainty creates greater computational complexity. To deal with this, a more specific type of fuzzy set, called the interval type-2 fuzzy set, can be used to reduce this complexity (Liang and Mendel, 2000).

Automatic methods facilitate extraction of knowledge from databases and can help with discovery of the ideal parameters, thereby avoiding the need for exhaustive simulations to determine the best parametrization for the model. Methods for discovery of knowledge in large databases for modeling or identification of fuzzy systems are therefore desirable (Sala et al., 2005; Cintra, 2012).

With regard to type-1 fuzzy sets, there are several proposals in the literature for discovering membership functions from data. However, the authors of previous reviews of the subject (Cintra et al., 2008; Medasani et al., 1998) have found that the methods investigated are complex and there is a lack of criteria on which to base the choice of which best fits the problem.

It is against this background that this paper presents a review of the current state of the art in development of type-1 and type- 2 membership functions. Overall, it is clear that there are still very few studies that have dealt with construction of type- 2 sets, while optimization methods are widely used for type-1 sets. Table 1 contains a summary of the characteristics of the methods reviewed for generation of type-1 membership functions. All of these methods are developed from the starting point of a database containing information about the problem domain. Some of the types of problems that are explored by each method include controllers, data mining, image processing, pattern recognition and classification.

The shape of membership functions is predetermined for all of the methods except for the method based on neural networks. The type most often used is the triangular function. Additionally, the only method in which the number of fuzzy sets defined for each membership function is not fixed is the histogram method. It should be pointed out that the characteristics listed in Table 1 are specific to the models described in the studies reviewed in this paper, which will be presented in greater detail in the sections that follow.

There is little published on methodology for constructing membership functions for type-2 fuzzy sets and this is therefore a fertile field for research (Mendel, 2010). The subject is also increasing in importance because of the growing number of applications for type2 functions (Wu and Mendel, 2014).

Optimization algorithms are often used to define type-1 membership functions and they can also be used for definition of type- 2 membership functions. However, in this case they are only able to explore a restricted number of parameters, because of the computational complexity involved (Linda and Manic, 2011). In general, the studies reviewed in this paper employ heuristics, histograms, C-means algorithms, simulated annealing and/or statistical data for definition of type- 2 set membership functions, as summarized in Table 2 .

It was observed that the majority of the methods reviewed do not concern themselves with transparency, i.e., with the interpretability of the sets that are generated. Additionally, the shapes of the functions are not discovered as part of this process, rather they are chosen a priori. Some of these methods employ data during the process of function definition, while others employ domain experts.

Table 1. Characteristics of methods for generation of type-1 membership functions

\begin{tabular}{|c|c|c|c|c|}
\hline \multirow[b]{2}{*}{ Methods } & \multicolumn{4}{|l|}{ Characteristics } \\
\hline & Source of knowledge & Domains & $\begin{array}{l}\text { Shape of the } \\
\text { membership functions }\end{array}$ & $\begin{array}{l}\text { Number of } \\
\text { fuzzy sets }\end{array}$ \\
\hline Neural networks & Data & Classification & Variable & Fixed \\
\hline Histogram & Data & Pattern recognition, classification & Gaussian & Variable \\
\hline Genetic algorithms & $\begin{array}{l}\text { Data and experts on the } \\
\text { problem domain }\end{array}$ & Controllers & Triangular, trapezoidal & Fixed \\
\hline Ant colony & Data & Data mining, controllers & Triangular, variable & Fixed, variable \\
\hline particle swarm & $\begin{array}{l}\text { Data and experts on the } \\
\text { problem domain }\end{array}$ & $\begin{array}{l}\text { Controllers, image } \\
\text { processing }\end{array}$ & $\begin{array}{l}\text { Gaussian, triangular, } \\
\text { s-shaped }\end{array}$ & Fixed \\
\hline Others & Data & Classification, controllers & Triangular, Gaussian & 2 to 9 sets \\
\hline
\end{tabular}


Table 2. Characteristics of methods for generation of type-2 membership functions

$$
\text { Characteristics }
$$

\begin{tabular}{|c|c|c|c|c|}
\hline Methods & Source of knowledge & Domains & $\begin{array}{l}\text { Shape of the } \\
\text { membership functions }\end{array}$ & $\begin{array}{l}\text { Number of } \\
\text { fuzzy sets }\end{array}$ \\
\hline $\begin{array}{l}\text { Linda and Manic } \\
\text { (2011) }\end{array}$ & $\begin{array}{l}\text { Data and experts on } \\
\text { the problem domain }\end{array}$ & Sensors & Gaussian & Fixed \\
\hline Mendel (2007b) & $\begin{array}{l}\text { Experts and non-experts } \\
\text { on fuzzy systems }\end{array}$ & Any & All types & Undefined \\
\hline $\begin{array}{l}\text { Choi and Rhee } \\
\text { (2009) }\end{array}$ & $\begin{array}{l}\text { Data and experts on } \\
\text { the problem domain }\end{array}$ & Pattern recognition & All types & Fixed and variable \\
\hline Almaraashi (2012) & Data & Prediction of temporal series & Gaussian & Fixed \\
\hline Hidalgo et al. (2012) & Data & Biometrics & Gaussian & Fixed \\
\hline Maldonado et al. (2014) & Data & Controllers & Triangular and trapezoidal & Fixed \\
\hline
\end{tabular}

Methods for generation of type-1 and type-2 membership functions will be presented in detail in Sections 2 and 3 of this article. Section 4 ends the paper with the Conclusions.

\section{Type-1 Fuzzy Sets}

Fuzzy modeling is an important approach that is widely used in applications in which the domain is characterized by uncertainty or by imprecise information. Applications of fuzzy modeling can be found in fields such as pattern recognition and classification (Das, 2013; Bombardier and Schmitt, 2010; Al-Sakka et al., 2013), controllers (Karthikeyan et al., 2013), simulation (Abd et al., 2013), image processing (Kaur and Sethi, 2013) and others.

By constructing these applications employing fuzzy set theory, it is possible to build a mechanism that is capable of representing the approximate reasoning that human beings engage in when faced with uncertainty and can also be used as a tool for representing the terms used in natural language (Ross, 2010). This is because, in contrast with classic set theory, in which the elements either are or are not members of a given set, a fuzzy set has the ability to represent the degrees of membership of elements of a universe of discourse. This makes a fuzzy set a powerful tool for representing the uncertainty and vagueness of linguistic terms (Klir and Yuan, 1995).

The Equation 1 (Klir and Yuan, 1995) represents the mapping of degrees of membership of elements in universe $X$ onto a fuzzy set $\mathrm{A}$, which is also known as a type-1 fuzzy set. This notation signifies that each element $x$ in universe $X$, does not simply belong or not to fuzzy set A, rather it has a degree of membership of fuzzy set A that falls within an interval from zero to 1 :
$\mu_{A}: X \rightarrow[0,1]$

The membership function for fuzzy set A can be written as in Equation 2 (Zadeh, 1965), which is a representation that is adequate for a continuous domain:

$A=\left\{\int \frac{\mu_{A}(x)}{x}\right\}$

In order to be in a position to define the fuzzy set for the domain of a problem, it is necessary to plan the concepts that will be dealt with in the problem in such a way that they adequately reflect the knowledge, whether this is acquired from data or from experts. With regard to this, it is necessary, in addition to mapping the degrees of membership, to also determine the shape of the fuzzy set's membership function. Commonly used shapes include: Triangular, Trapezoidal, Gaussian, S-shaped and Z-shaped. The shape of the membership function also has an impact on the results and so the function that best fits the representation of the fuzzy set should be chosen.

Figure 1 illustrates the elements of a fuzzy set. A normal fuzzy set will have at least one element that has a membership value equal to one, which is shown in the center, or core. There are also elements that have degrees of membership that fall within the interval from 0 to 1 , which is an area that has been termed the boundary. All elements $x$ of universe $X$ that are part of fuzzy set $A$ are distributed within a region called the support (Ross, 2010).

Therefore, definition of a membership function must take account of the following: (a) how many fuzzy sets will be defined for the variable, (b) what shapes the functions have and (c) which parameters will be adopted for the membership function. 


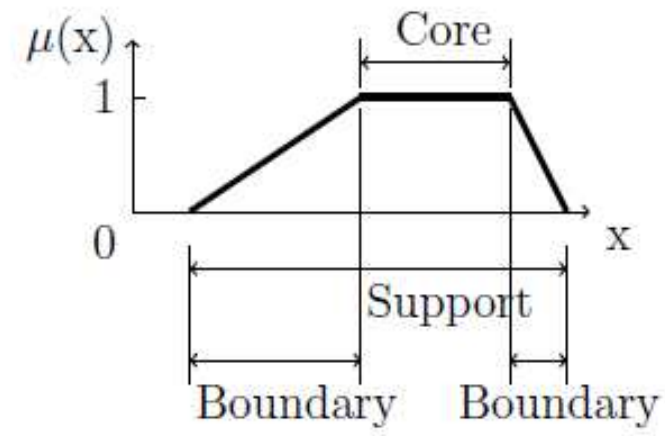

Fig. 1. Elements of a fuzzy set. Source: Ross (2010)

\section{Automatic Methods for Generation of Type- 1 Membership Functions}

Although definition of membership functions is an important step in modeling fuzzy systems, there is no predefined sequence of steps to be followed. Additionally, the methods that have been described in the literature are restricted to specific domains of application (Cintra et al., 2008; Medasani et al., 1998).

Calling on the help of experts on the domain of the problem is a widely-employed technique. Very often, however, the complexity of the problem to be dealt with and the number of variables involved mean that the task is onerous. The alternative is to choose a method for automatization of development of membership functions. The subsections that follow will present some of these methods, based on neural networks, heuristics, histograms, genetic algorithms, ant colonies, particle swarms and other techniques.

\section{Neural Networks}

Neural networks are one way of acquiring degrees of membership. The method requires both a training dataset (to enable the network to learn from examples) and a dataset for generating the membership functions. In a neural network designed for classification, the conventional architecture takes the characteristics of the problem as input neurons and the problem classes as output neurons (Ross, 2010). The membership function that results from this proposal is illustrated in Fig. 2.

It can be observed from Fig. 2 that the result of this neural network is a given element's degree of membership of the regions of the problem, which are R1, R2 and R3.

Yang and Bose (2006) performed the generation of membership functions using self-organizing maps and applied the method to a classification problem. In this model the network input vector is composed both of the problem characteristics and of the problem classes. According to the authors, this approach offers advantages over other methods when there are imbalances in samples.

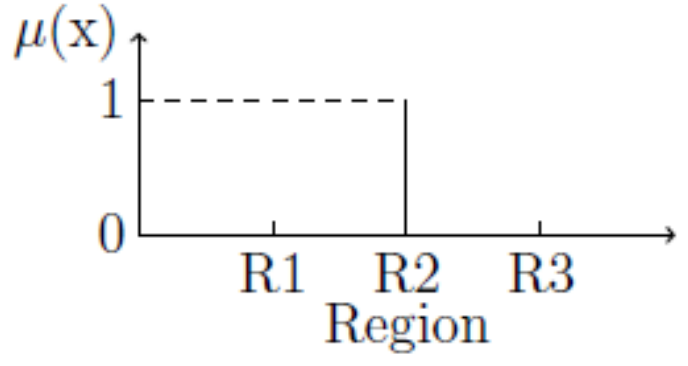

Fig. 2. Membership function for one point $(\mathrm{x} 1, \mathrm{x} 2)$ in the problem domain. Source: Ross (2010)

Other methods that were investigated in that study exhibited variations in the shape of the membership function when the data contained noise (the Histogram and Fuzzy C-Means methods).

\section{Histogram}

A histogram represents the frequency distribution of a variable. In statistics, a histogram is used to understand the scenario of which the variable whose distribution it represents is a part. It is this property that has enabled researchers to explore methods for using a histogram to define membership functions.

Medasani et al. (1998) proposed a method for definition of membership functions for each class or region of a problem using characteristics histograms. To achieve this, histograms must be obtained for each characteristic of the class or region and then a Gaussian distribution mixture model must be found that represents the weighted sum of the densities of the characteristics of the problem (Reynolds, 2008). The resulting distribution will then be used as the membership function. The authors who describe this technique consider it simple to implement.

A study published by Choi and Rhee (2009) investigating fuzzy pattern recognition proposed a strategy for obtaining membership functions in which histograms for the classes in the problem are smoothed using a method called 3 point Triangular window. Additionally, the histogram is also normalized so that it can be fitted to a polynomial function (of the minimum possible degree). In that study specifically, the histogram was fitted to a Gaussian function. The total number of Gaussian functions depends on the peaks that are obtained from the histogram and the mean of the Gaussian functions will be located at the apices of the peaks of the histogram. The procedure proposed is not considered complex.

\section{Genetic Algorithms}

A genetic algorithm (Holland, 1975) is an evolutionary method that has been widely adopted and 
employed for problems of optimization. The algorithm develops a strategy for generation of populations that is always based on the best individuals from previous generations. At the end of the algorithm's execution, it is obtained a population with the fittest individuals (solutions) in accordance with the objective function of the method. Methods based on genetic algorithms are widely used for generation of membership functions. Herman et al. (2009) developed a genetic algorithm to find the membership functions for a fuzzy controller. The algorithm evaluates the membership functions of the population based on the overshoot (the percentage that exceeds the variable's final values) of the response. Each individual in the population represents all of the membership functions, both input and output and each gene in the chromosome is a linguistic variable in the problem. Each membership function has five fuzzy sets, three Triangular and two Trapezoidal. Additionally, the parameters are real numbers. The results observed in that study were considered satisfactory for the problem in question (Herman et al., 2009).

Figure 3 illustrates the structure usually employed for genetic algorithms for optimization of fuzzy membership functions. Each chromosome in the solution presents the codification of the parameters of all of the membership functions, both input and output. Each element shown in the definition of Input 1 represents the parameter of a specific membership function $\left(\mathrm{MF}_{1} \mathrm{P}_{1}\right.$-parameter 1 of membership function 1). In the case of Fig. 3, the definition of Input 1 has $n$ membership functions, and trapezoidal functions are represented at the start and the end of the string.

A paper published by Huynh et al. (2012) presents a proposal in which different shapes of membership functions are employed. The parameters of the membership functions are each adjusted individually and the functions can take asymmetrical shapes. The result was improved interpretability of the rule base through a process of reducing the number and length of the rules for the fuzzy system for which the method was tested.

\section{Ant Colony}

The Ant Colony System is a method inspired on the behavior of ant colonies. It is an algorithm for search and optimization according to an objective function, which can either be minimization or maximization (Colorni and Dorigo, 1991) (Dorigo et al., 1996).

Hong et al. (2009) applied the Ant Colony System algorithm to extraction of membership functions in a fuzzy data mining problem. Each membership function has discreet parameters and is represented by a string of bits and each membership function represents one possible solution within the search space.

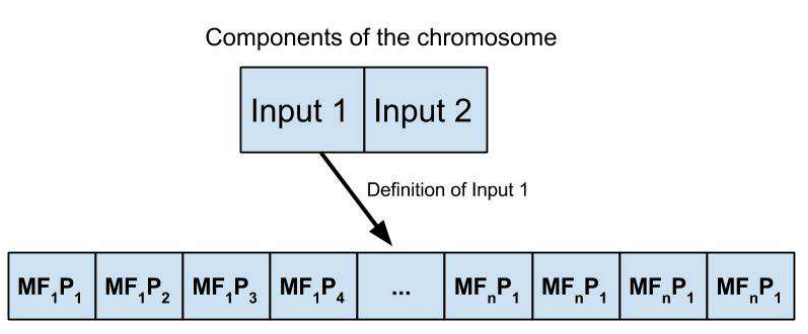

Fig. 3. Chromosomal structure of a genetic algorithm for optimization of membership functions

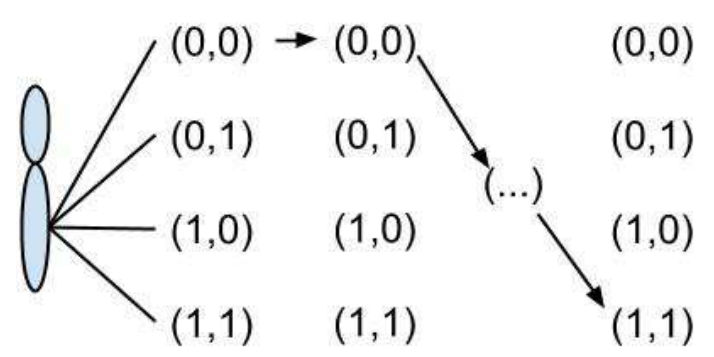

Fig. 4. The solution found by an ant. Source: adapted from Hong et al. (2009)

At the end of execution of the algorithm, the membership function with the highest fitness value is chosen, i.e., the function closest to the objective function (and which corresponds to the route followed by the ant that had the greatest quantity of pheromone). The membership functions produced in that study are triangular and are defined by 2 parameters, which indicate the center of the triangle and its width. Initially, the membership function parameters are set to values that include the interval of the items in the database. Graphs are plotted to identify the path to the best set of membership functions.

Figure 4 illustrates an example of the graph plotted during execution of the algorithm. In this graph, each point that is plotted will be used to define the width and center of a triangular membership function (Hong et al., 2009).

A later paper by $\mathrm{Wu}$ et al. (2012) described an improved method for discovery of membership functions in the domain of fuzzy data mining in which the parameters can be continuous. Additionally, the adapted version generates the solution discovery graph dynamically. The authors stated that the results achieved were of higher quality than those reported in the earlier paper by Hong et al. (2009), in which the parameters had been defined in a discrete space.

Castillo et al. (2015), the authors proposed a modified ACO based on five of its variations: Ant System (AS), Elitist Ant System (EAS), Rank Based Ant System (ASRank), Man-Min Ant System (MMAS), Ant Colony System (ACS). These variations of the ACO are applied sequentially to optimize the shape of the MF 
(Triangular, Trapezoidal, Gaussian, etc.) and its parameters, and the rules of the fuzzy system. The algorithm was applied to a ball and beam system. The results obtained by the proposed method were satisfactory when comparing to each variation applied individually.

\section{Particle Swarm}

Particle Swarm Optimization (PSO) is based on the behavior of groups of individuals, such as birds or fish, for example. In this type of algorithm, the individual members of a population learn from their own experiences and also from the experiences of the individuals that exhibit the best performance in the group. This property can allow the group to successfully locate its goal (Kennedy and Eberhart, 1995). There are a number of publications that describe development of membership functions based on particle swarm algorithms (Nieradka and Butkiewicz, 2007; Fang et al., 2008; Permana and Hashim, 2010).

Nieradka and Butkiewicz (2007) conducted a study employing a PSO algorithm to optimize the parameters of fuzzy membership functions. Their research was in the area of image processing and the purpose was to model linguistic concepts used in the area. The membership functions parameters were optimized using the concepts of entropy and a fuzzy index. The function that best fits the problem is the one with greatest entropy (the greatest quantity of information) and has the greatest vagueness index (the function with the highest index is that which best describes the fuzzy set). The format chosen for the sets was S-shaped.

Fang et al. (2008) developed a fuzzy controller to control water level in a water tank. The particle swarm method was used to find parameters of a membership function with a pre-established shape. Thus, the particle represents the input and output parameters of the function. The objective is to minimize the control's error, tuning the membership functions as necessary. The method proved to be adequate when compared with a conventional PDI controller. However, the membership functions had subnormal shapes (there were sets with no elements with a degree of membership equal to 1) and only Gaussian functions were used.

In research by Permana and Hashim (2010), the particle swarm algorithm was used to optimize the response of a controller for the Backup trucker problem, in which the intention is to guide a wagon from any point so that it arrives at the dock perpendicularly. Both input and output membership functions have a triangular shape. The PSO algorithm is used to optimize three parameters of these functions: center, right and left. The membership functions are tuned using the algorithm until the performance of the fuzzy system is considered adequate. The number of fuzzy sets for the membership functions was determined on the basis of existing knowledge about the Backup trucker problem, for which linguistic terms are already known. The results achieved with this method were superior in terms of response and convergence, when compared to a fuzzy system defined at the start on the basis of prior knowledge (Permana and Hashim, 2010).

\section{Methods Based on Heuristics}

Finally, there are also strategies described in the literature employing heuristics to investigate definition of membership functions. Two such heuristic approaches, applied to pattern recognition problems, are: FuzzyDBD (Cintra et al., 2009) and FuzzyDBDII (Cintra et al., 2011).

The FuzzyDBD method (Cintra et al., 2009) was considered a rapid method by its authors. Working from a database, the result of the method is the membership functions for the problem. In order to achieve this purpose, the membership function is defined as triangular, the total number of fuzzy sets for each variable is determined using the Wang and Mendel method (Wang, 2003) and the fuzzy sets are equally distributed across the problem domain (all sets have the same sized support). The steps followed in that study are summarized in Fig. 5.

With the aim of not always generate the same number of fuzzy sets for all attributes, Cintra et al. (2011) improved FuzzyDBD to create FuzzyDBDII. In the later study each attribute was evaluated individually to determine the total number of sets needed. Three different techniques to achieve this end were assessed: gain information, ReliefF ranking filter, and the Wang and Mendel method. These techniques were analyzed using a FuzzyDT, which is a fuzzy decision tree. Experiments were conducted using a database for classification problems and the proposed method exhibited promising results (Cintra et al., 2011). The authors concluded that the interval of 2 to 9 fuzzy sets per problem attribute should have been restricted further in order to avoid generating large rule bases.

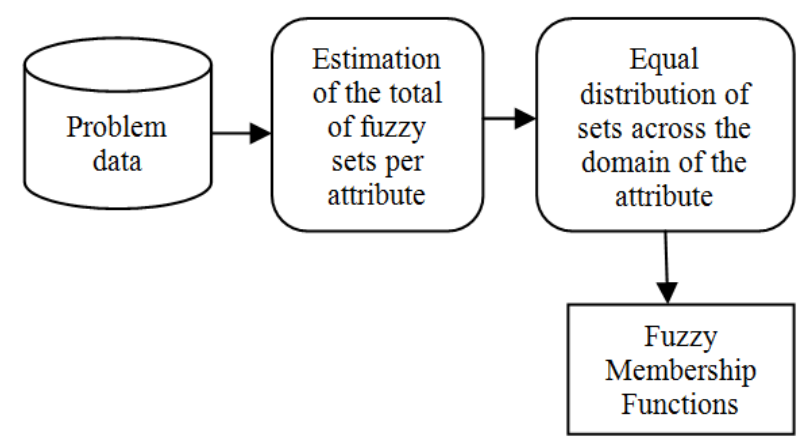

Fig. 5. Steps in implementation of the Fuzzy DBD method. Source: Cintra et al. (2009) 
Another interesting research is related to hybrid methods. Martínez-Soto et al. (2015), the authors developed a hybrid GA-PSO to solve the minimization of the steady state error in two types of controllers, with different levels of complexity. The results obtained by the hybrid method were better than those achieved by the application of PSO and GA individually.

\section{Considerations on Methods for Generation of Type- 1 Membership Functions}

All of the methods reviewed and described above were applied to specific domains and are not therefore generic approaches. Furthermore, each of them has its own problems that are dependent on the domain of application. In the case of neural networks, for example, these require training data and can be complex to implement, but they can be a good option for nonlinear problems.

The histogram technique can be implemented when problems have a significant quantity of data. However, whether or not the quantity of data is significant will only be known when the method is implemented.

Genetic algorithms, the ant colony system and particle swarms all require an objective function to be able to find a solution. Additionally, they can become stuck at local maximums (minimums) and can also take a long time to converge. Heuristic methods have provided good solutions with low computational complexity, with restriction of the domain of application. Additionally, the heuristic methods described in this article offer a more wide ranging solution for design of fuzzy systems, including with regard to the rule base.

\section{Type-2 Fuzzy Sets}

Type-2 fuzzy sets were introduced by Zadeh (1975), who created them as a method for dealing with uncertainty related to construction of the membership functions themselves, allowing degrees of membership to be represented not by crisp numbers, but by another fuzzy set. However, until the 1990s the volume of research in this area was small, although it has increased since then (Mendel, 2007a; John and Coupland, 2007). As the field matured, two classes of type-2 sets have coalesced:

- General type-2 fuzzy sets: these are sets for which the degree of membership is also a fuzzy set. Thus, fuzzification of element $x$ in the primary domain has a degree of membership $u$ which, in turn, has a degree of membership, known as secondary degree of membership. Mathematically, the definition of a general type-2 fuzzy set can be expressed by Equation 3:

$$
\tilde{A}=\left\{(x, u), \mu_{\tilde{A}} \mid \forall x \in X, \forall u \in J_{x}, J_{x} \subseteq[0,1]\right\}
$$

Where:

$X=$ Primary domain

$J_{x}=$ Secondary domain, and the values of both vary within the range 0 to 1

- Interval type-2 fuzzy sets (Liang and Mendel, 2000): these are sets in which the secondary degree of membership always has the value 1 . Therefore, in fuzzification of element $x$, the value of $u$ is found, which, in turn, reaches 1 . This means that the combination of input $x$ and the primary degree of membership $u$ always result in the same secondary degree. The definition of an interval fuzzy set is expressed in Equation 4:

$\tilde{A}=\left\{(x, u), 1 \mid \forall x \in X, \forall u \in J_{x}, J_{x} \subseteq[0,1]\right\}$

Where:

$X=$ Primary domain

$J_{x}=$ Secondary domain, and the values of both vary within the range 0 to 1

The primary uncertainty of a type-2 fuzzy set can illustrated as its Footprint of Uncertainty (FOU), whereby the greater the footprint, the greater the uncertainty (Mendel, 2003). In the case of type-2 interval sets, the FOU completely describes the uncertainty present in the fuzzy set.

Figure 6 shows three different shaped footprints of uncertainty. The footprint of uncertainty of a type- 2 function is delimited by a lower membership function and by an upper membership function. Example (a) in Fig. 6 illustrates a Gaussian type-2 fuzzy function with uncertainty at the standard deviation, which means that there are a range of values for the standard deviation. Note that the mean is the same for the upper and lower functions. Figure $6 \mathrm{~b}$ illustrates a footprint of uncertainty formed by two functions with differing means and standard deviations. The example in Fig. 6c illustrates a sigmoidal function with inflection uncertainty, indicating that the functions have different inclines. The greater the difference between the upper and lower functions, the larger the footprint of uncertainty, which denotes greater uncertainty. The FOU is expressed by Equation 5:

$\operatorname{FOU}(\tilde{A})=\bigcup_{x \in X} J_{x}$ 


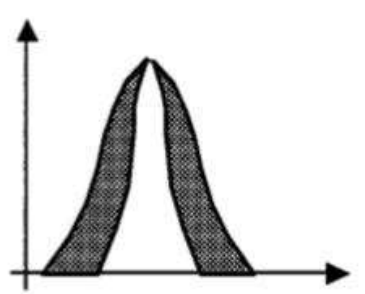

(a)

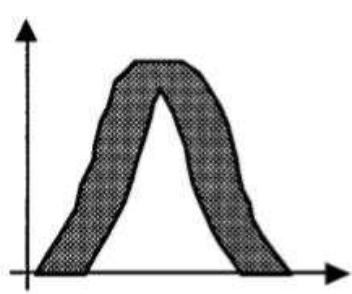

(b)

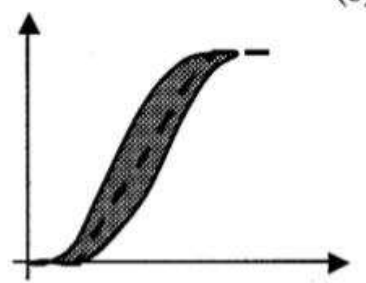

(c)

Fig. 6. Footprints of Uncertainty: (a) Gaussian Function with uncertain standard deviation; (b) Gaussian Function with uncertain mean; (c) Sigmoidal Function with inflection uncertainties. Source: Mendel and John (2002)

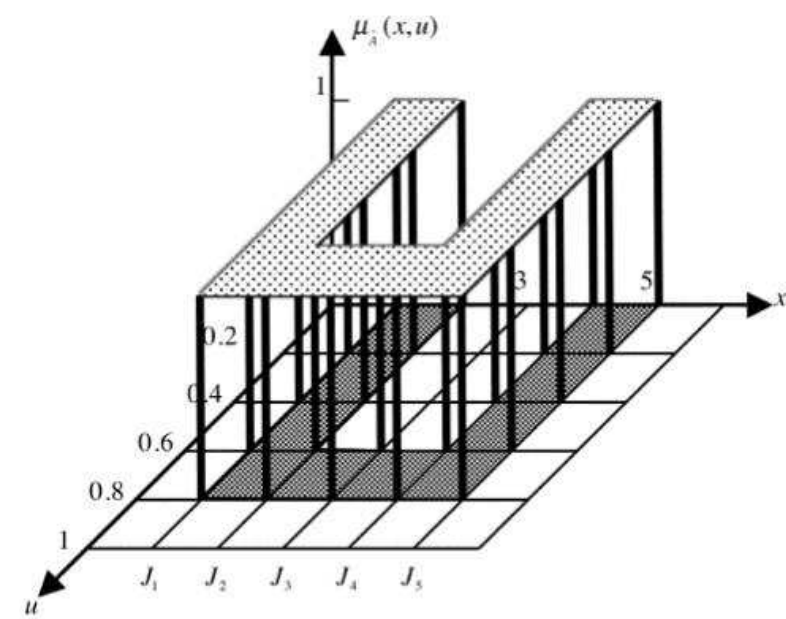

Fig. 7. Interval type-2 membership function in a discrete universe. Source: Mendel et al. (2006)

In Equation 5, $X$ represents the set of all elements in the problem domain and $J_{x}$ represents all primary degrees of membership. The footprint of uncertainty of a fuzzy set is therefore the union of all primary degrees of membership. The FOU is delimited by two membership functions, called the upper membership function and the lower membership function. Figure 7 shows a three-dimensional illustration of an interval type-2 membership function.

It can be observed from Fig. 7 that the area under the $x$ and $u$ axes represents the FOU (Mendel et al., 2006). An image of the FOU is reflected along the secondary degree of membership axis because all of its elements have degrees of membership of 1 .
There are currently a number of tools and procedures for development of type-2 fuzzy systems, but the number is still limited. Two such solutions were developed for Matlab ${ }^{\circledR}$ software (Karnik et al., 2008; Castro et al., 2007). The first is a set of procedures for conducting operations on type-2 fuzzy sets and can be obtained free of charge. Both procedures for operations on type- 1 and type-2 sets are available. The second implementation for Matlab (Castro et al., 2007) offers algorithms for executing operations on interval type- 2 sets and has a similar interface to that of the well-known "Fuzzy Logic Toolbox" for Matlab ${ }^{\circledR}$. A more recent solution is a framework developed on the Java platform (Wagner, 2013), created with the objective of facilitating access to and widening use of type-2 fuzzy systems. This tool implements type-1 and type-2 fuzzy inference systems and can be obtained free of charge. The authors recently made a web-based interface (Wagner et al., 2014).

\section{Methods for Generation of Type-2 Membership Functions}

Bearing in mind how long it is since type-2 fuzzy sets were first proposed by Zadeh (1975), their use remains restricted (Mendel, 2007a). Nevertheless, they have been successfully used in several applications, such as: Controllers (Sepúlveda et al., 2012; Chaoui et al., 2013), industrial applications (Dereli et al., 2011), data mining (Turksen and Celikyilmaz, 2010), image processing and pattern recognition (Melin, 2012; Mitchell, 2005) and in a predictive model for financial applications (Bernardo et al., 2013). In terms of approaches to construction of the membership functions, in the majority of the studies each author develops functions employing the available knowledge (which is dependent on the domain of the application), heuristically or with the help of experts.

The type-2 fuzzy set approach has proven appropriate in applications involving data that vary over time and data containing noise or when it is necessary to extract information from an expert (Mendel, 2004).

Although type- 2 membership functions offer satisfactory results for domains involving uncertainty, they also involve greater computational complexity because of the type reduction stage. For this reason, the majority of applications employ interval type- 2 sets (Mendel, 2004). Interval type-2 sets require less complex calculations and apply the same types of calculations employed for type-1 sets (Mendel et al., 2006).

Techniques for defining type-2 fuzzy systems have been receiving growing research interest in response to the promising results and the consequent increase in use. This is because definition of membership functions is an important stage in the process of developing these systems and one that has a strong impact on the results. However, few studies have focused on development of type-2 membership functions. 
When input membership functions are adequately constructed and are compatible with a representation of the uncertainty that is present, then the system's output will also adequately represent uncertainty (Linda and Manic, 2011). A study by Linda and Manic (2011) attempted to achieve this, focusing on development of interval type-2 functions in which the FOU is characterized by the standard deviation of the data.

A study by Mendel (2007a) proposed methodology for development of interval type-2 functions for words, in which knowledge is extracted from experts on the subject of fuzzy systems; and also proposed methodology for knowledge extraction from people who are not experts in fuzzy systems. The data are aggregated with equal weighting given to data from many different individuals and, because of this, the author suggests that the technique could be called fuzzistics, since it also combines statistics.

Choi and Rhee (2009) focused on problems of pattern recognition, presenting three methods for obtaining the FOU for interval type-2 fuzzy sets: heuristics, histograms and C-means. In the heuristic approach, a type-1 membership function shape is chosen to best fit the problem and then the lower membership function for the FOU is found by multiplying the first by a constant from 0 to 1 . This method is conducted with the help of experts on the subject. The same authors then propose a solution based on histograms. In this case, histograms for the characteristics of the problem are needed. These are then smoothed and normalized to obtain the minimum degree polynomial function possible. The total number of fuzzy sets and their heights are determined by this polynomial function. The technique employing the C-means algorithm utilizes two equations to define the upper and lower membership functions, rather than the single equation used in the standard method. However, although all three methods proved satisfactory, the authors themselves wonder whether the resulting membership functions may not correctly represent the uncertainty present in the data and considered the study as a starting point for developing improved methods.

The approach adopted by Hidalgo et al. (2012) was to start with an existing type- 1 system and then, with the help of a genetic algorithm, they optimized the parameters. Optimization of parameters was conducted in three different ways, applying three different levels of uncertainty. At the first level, the footprint of uncertainty was the same size for all inputs. In the second treatment, each input could have different FOUs and in the third, each input was tested with different FOUs for its own sets. At the end of execution, the system that best fits the problem is chosen.

Almaraashi (2012) published a study employing an optimization method that had not previously been used to determine type-2 membership functions, Simulated Annealing. The initial optimized sets are randomly generated and then the Simulated Annealing algorithm is executed to find the best combination of parameters, the optimum combination. Although this author reported promising results using type- 2 sets, the complexity of the algorithm and the time required were significant.

Maldonado et al. (2014) performed a multiobjective genetic optimization of an AT2-FIS. An AT2-FIS is an Interval Type-2 Fuzzy Inference System obtained by means of two averaged T1-FIS. The system was developed in hardware, more specifically in a FPGA (Field-Programmable Gate Array). The goal of the work was controlling the speed of a direct current motor. The results obtained by the system (error rate and runtime) were satisfactory even when facing external interference.

\section{Considerations on Automatic Methods for Generation of Interval Type-2 Functions}

The greater part of the methods reviewed do not attach significance to the membership function's Footprint of Uncertainty (FOU), i.e., they do not explain the origin of the uncertainty in the data of the problem. Rather, these applications define a footprint of uncertainty that enables system to achieve results appropriate to the context. However, Linda and Manic (2011) did employ the standard deviation of the data as a link to the uncertainty of the membership functions. Similarly, Mendel's approach (2007a) is naturally transparent with regard to the FOU, because it involves people in the process of defining the membership functions, but it is not an automatic method. An automatic method may take into consideration the transparency of the membership functions, which is an appreciable characteristic of a fuzzy inference system (Riid, 2002; Chen and Chen, 2007). In this regard, some restrictions could be implemented, such as in Castellano et al. (2003). Furthermore, it must be considered if the desired results are achieved by the system. Thus, the balance between these features should be performed by the designers and experts, depending on the importance of each of these characteristics to the problem addressed.

With regard to the domains of application of these methods, the range is varied, but each method is developed for a specific domain, such as, for example, pattern recognition. The exception is a study published by Mendel (2007a), which was an approach to modeling words and can therefore be applied to any domain in which this type of modeling is necessary.

The absence of arguments to justify the choice of membership function shape, which in the majority of cases is Gaussian, is worth noting. Even in methods 
that used other types of membership functions, the shapes were chosen before execution of the method. Along the same lines, the number of sets used for each variable is fixed before the procedure proposed is applied. Wu and Mendel (2014) make some suggestions about configuration of type-1 and type- 2 fuzzy systems in a recent publication. For example, they suggest using Gaussian or trapezoidal functions to define type- 2 sets.

\section{Conclusion}

This paper has presented a review of methods for generation of type-1 and type-2 membership functions. Type-1 membership functions have existed for longer and as a result are the subject of a greater number of studies. In general, type-1 membership functions are defined using parameter optimization methods. As has been observed in previous studies (Medasani et al., 1998; Cintra et al., 2008), these mechanisms are complex and application dependent. The heuristic approach may offer advantages over the other methods because of reduced complexity, but it is necessary to evaluate whether the results returned will be satisfactory.

With regard to interval type-2 sets extracted from data, there is not yet a generalized method or an established methodology. Since type- 2 sets are employed for representation of uncertainty, it is necessary that the automatic methods improve in their ability to represent the FOU (footprint of uncertainty) in a manner that reflects this uncertainty. Acquisition of information from domain experts is already at a more advanced stage, with recommendations of a series of steps to be followed to define the fuzzy membership functions.

\section{Author's Contributions}

All authors equally contributed in this work.

\section{Ethics}

The corresponding author confirms that all of the other authors have read and approved the manuscript and no ethical issues involved.

\section{References}

Abd, K., K. Abhary and R. Marian, 2013. Development of a fuzzy-simulation model of scheduling robotic flexible assembly cells. J. Comput. Sci., 9: 1761-1768.

DOI: $10.3844 /$ jcssp.2013.1761.1768

Almaraashi, M., 2012. Learning of type-2 fuzzy logic systems using simulated annealing. Ph.D. Thesis, De Montfort University, Leicester, UK.
Al-Sakka, H., A.A. Boumahmoud, B. Fradon, S.J. Frasier and P. Tabary, 2013. A new fuzzy logic hydrometeor classification scheme applied to the french $\mathrm{x}-$, c- and s-band polarimetric radars. J. Applied Meteor. Climatol., 52: 2328-2344.

DOI: 10.1175/JAMC-D-12-0236.1

Bernardo, D., H. Hagras and E. Tsang, 2013. A genetic type-2 fuzzy logic based system for the generation of summarised linguistic predictive models for financial applications. Soft Comput., 17: 2185-2201. DOI: $10.1007 / \mathrm{s} 00500-013-1102-y$

Bombardier, V. and E. Schmitt, 2010. Fuzzy rule classifier: Capability for generalization in wood color recognition. Eng. Applic. Artificial Intellig., 23: 978-988. DOI: 10.1016/j.engappai.2010.05.001

Castellano, G., A.M. Fanelli and C. Mencar, 2003. Design of Transparent Mamdani Fuzzy Inference Systems. In: Design and Application of Hybrid Intelligent Systems, Abraham, A., M. Köppen and K. Franke (Eds.), IOS Press, Amsterdam, ISBN-10: 1586033948, pp: 468-476.

Castillo, O., E. Lizárraga, J. Soria, P. Melin and F. Valdez, 2015. New approach using ant colony optimization with ant set partition for fuzzy control design applied to the ball and beam system. Inf. Sci., 294: 203-215. DOI: 10.1016/j.ins.2014.09.040

Castro, J.R., O. Castillo and L.G. Martínez, 2007. Interval type2 fuzzy logic toolbox. Eng. Lett., 15: 89-98.

Chaoui, H., W. Gueaieb, M. Biglarbegian and M.C.E. Yagoub, 2013. Computationally efficient adaptive type-2 fuzzy control of flexible-joint manipulators. Robotics, 2: 66-91. DOI: 10.3390/robotics2020066

Chen, L. and C.L.P. Chen, 2007. Pre-shaped Fuzzy CMeans algorithm (PFCM) for transparent membership function generation. Proceedings of the IEEE International Conference on Systems, Man and Cybernetics, Oct. 7-10, IEEE Xplore Press, Montreal, pp: 789-794. DOI: 10.1109/ICSMC.2007.4413722

Choi, B.I. and F.C.H. Rhee, 2009. Interval type-2 fuzzy membership function generation methods for pattern recognition. Inform. Sci., 179: 2102-2122. DOI: 10.1016/j.ins.2008.04.009.

Cintra, M.E., H.A. Camargo and M.C. Monard, 2008. A study on techniques for the automatic generation of membership functions for pattern recognition. Congresso da Academia Trinacional de Ciências.

Cintra, M.E., H.A. Camargo and T.P. Martin, 2009. Optimising the fuzzy granulation of attribute domains. Proceedings of the International Fuzzy Systems Association World Conference, (AWC' 09), Lisboa, Portugal, pp: 742-747.

Cintra, M.E., M.C. Monard, E.A. Cherman and H.A. Camargo, 2011. On the estimation of the number of fuzzy sets for fuzzy rule-based classification systems. Proceedings of the 11th International Conference on Hybrid Intelligent Systems, Dec. 5-8, IEEE Xplore Press, Melacca, pp: 211-216. DOI: $10.1109 / \mathrm{HIS} .2011 .6122107$ 
Cintra, M.E., 2012. Genetic generation of fuzzy knowledge bases: New perspectives. Ph.D. Thesis, University of São Paulo (USP), São Carlos, SP.

Colorni, V.M.A. and M. Dorigo, 1991. Distributed optimization by ant colonies. Proceedings of the 1st European Conference on Artificial Life, (ECA' 91), Paris, France, pp: 134-142.

Das, S., 2013. Pattern recognition using the fuzzy c-means technique. Int. J. Energy Inform. Commun., 4: 1-14.

Dereli, T., A. Baykasoglu, K. Altun, A. Durmusoglu and I.B. Türksen, 2011. Industrial applications of type-2 fuzzy sets and systems: A concise review. Comput. Industry, 62: 125-137. DOI: $10.1016 /$ j.compind.2010.10.006

Dorigo, M., V. Maniezzo and A. Colorni, 1996. Ant system: Optimization by a colony of cooperating agents, Systems, Man and Cybernetics, Part B: Cybernetics. IEEE Trans., 26: 29-41.

DOI: $10.1109 / 3477.484436$

Fang, G., N.M. Kwok and Q. Ha, 2008. Automatic fuzzy membership function tuning using the particle swarm optimization. Proceedings of the Pacific-Asia Workshop on Computational Intelligence and Industrial Application, Dec. 19-20, IEEE Xplore Press, pp: 324-328. DOI: 10.1109/PACIIA.2008.105

Herman, N.S., I. Yusuf and S.M.B.H. Shamsuddin, 2009. Genetic algorithms and designing membership function in fuzzy logic controllers. Proceedings of the World Congress on Nature and Biologically Inspired Computing, Dec. 9-11, IEEE Xplore Press, Coimbatore, pp: 1753-1758.

DOI: 10.1109 /NABIC.2009.5393629

Hidalgo, D., P. Melina and O. Mendoza, 2012. Optimization method for membership functions of type-2 fuzzy systems based on the level of uncertainty applied to the response integration of modular neural network for multimodal biometrics. Proceedings of the Annual Meeting of the North American Fuzzy Information Processing Society, Aug. 6-8, IEEE Xplore Press, Berkeley, CA, pp: 1-6. DOI: $10.1109 /$ NAFIPS.2012.6291057

Holland, J.H., 1975. Adaptation in Natural and Artificial Systems: An Introductory Analysis with Applications to Biology, Control and Artificial Intelligence. 1st Edn., University of Michigan Press, Ann Arbor, ISBN-10: 0472084607, pp: 183.

Hong, T.P., Y.F. Tung, S.L. Wang, M.T. Wu and Y.L. $\mathrm{Wu}, 2009$. An ACS-based framework for fuzzy data mining. Expert Syst. Applic., 36: 11844-11852. DOI: $10.1016 / \mathrm{j}$.eswa.2009.04.016

Huynh, T., H. Nguyen and B. Le, 2012. A unified design for the membership functions in genetic fuzzy systems. Int. J. Comput. Sci., 9: 7-16.

John, R. and S. Coupland, 2007. Type-2 fuzzy logic: A historical view. IEEE Comput. Intellig. Magazine, 2: 57-62. DOI: 10.1109/ MCI.2007.357194
Karnik, N.N., Q. Liang, F. Liu, D. Wu and J. Joo et al., 2008. Type-2 fuzzy logic software.

Karthikeyan, R., K. Manickavasagam, S. Tripathi and K.V.V. Murthy, 2013. Neuro-fuzzy-based control for parallel cascade control. Chem. Product Process Modeling, 8: 15-25. DOI: 10.1515/cppm-2013-0002

Kaur, J. and P. Sethi, 2013. Evaluation of fuzzy inference system in image processing. Int. J. Comput. Applic., 68: 1-4. DOI: $10.5120 / 11708-7159$

Kennedy, J. and R. Eberhart, 1995. Particle swarm optimization. Proceedings of the IEEE International Conference on Neural Networks, Nov. 27 Dec. 1, IEEE Xplore Press, Perth, WA, pp: 1942-1948. DOI: $10.1109 /$ ICNN.1995.488968

Klir, G. and B. Yuan, 1995. Fuzzy Sets and Fuzzy Logic: Theory and Applications. 1st Edn., Prentice Hall PTR, Upper Saddle River, ISBN-10: 0131011715 , pp: 574.

Liang, Q. and J.M. Mendel, 2000. Interval type-2 fuzzy logic systems: Theory and design. IEEE Trans. Fuzzy Syst., 8: 535-550. DOI: 10.1109/91.873577

Linda, O. and M. Manic, 2011. Uncertainty modeling for interval type-2 fuzzy logic systems based on sensor characteristics. Proceedings of the IEEE Symposium on Advances in Type-2 Fuzzy Logic Systems, Apr. 11-15, IEEE Xplore Press, Paris, pp: 31-37. DOI: $10.1109 / \mathrm{T} 2 F U Z Z .2011 .5949550$

Maldonado, Y., O. Castillo and P. Melin, 2014. A multiobjective optimization of type-2 fuzzy control speed in FPGAs. Applied Soft Comput., 24: 1164-1174. DOI: 10.1016/j.asoc.2014.04.041

Martínez-Soto, R., O. Castillo, L.T. Aguilar, A.R. Díaz, 2015. A hybrid optimization method with PSO and GA to automatically design Type- 1 and Type- 2 fuzzy logic controllers. Int. J. Machine Learn. Cybernet., 6: 175-196. DOI: 10.1007/s13042-013-0170-8

Medasani, S., J. Kim and R. Krishnapuram, 1998. An overview of membership function generation techniques for pattern recognition. Int. J. Approximate Reason., 19: 391-417. DOI: $10.1016 / \mathrm{S} 0888-613 X(98) 10017-8$

Melin, P., 2012. Image Processing and Pattern Recognition with Mamdani Interval Type-2 Fuzzy Inference Systems. In: Combining Experimentation and Theory: A Hommage to Abe Mamdani, Trillas, E., P.P. Bonissone, L. Magdalena and J. Kacprzyk (Eds.), Springer Science and Business Media, Springer-Verlag New York Inc, ISBN-10: 3642246656, pp: 179-190.

Mendel, J.M. and R.I.B. John, 2002. Type-2 fuzzy sets made simple. IEEE Trans. Fuzzy Syst., 10: 117-127. DOI: $10.1109 / 91.995115$

Mendel, J.M., 2003. Type-2 fuzzy sets: Some questions and answers. IEEE Neural Networks Society Newsletter, 1: 10-13.

Mendel, J.M., 2004. Fuzzy sets for words: Why type-2 fuzzy sets should be used and how they can be used. IEEE-FUZZ tutorial. 
Mendel, J.M., R.I. John and F. Liu, 2006. Interval type-2 fuzzy logic systems made simple. IEEE Trans. Fuzzy Syst., 14: 808-821. DOI: 10.1109/TFUZZ.2006.879986

Mendel, J.M., 2007a. Advances in type-2 fuzzy sets and systems. Inform. Sci., 177: 84-110. DOI: $10.1016 /$ j.ins.2006.05.003

Mendel, J.M., 2007b. Computing with words and its relationships with fuzzistics. Inform. Sci., 177: 988-1006. DOI: 10.1016/j.ins.2006.06.008

Mendel, J.M., 2010. A quantitative comparison of interval type- 2 and type-1 fuzzy logic systems: First results. Proceedings of the IEEE International Conference on Fuzzy Systems, Jul. 18-23, IEEE Xplore Press, Barcelona, pp: 1-8. DOI: 10.1109/FUZZY.2010.5584727

Mitchell, H.B., 2005. Pattern recognition using type-II fuzzy sets. Inform. Sci., 170: 409-418. DOI: $10.1016 /$ j.ins.2004.02.027

Nieradka, G. and B. Butkiewicz, 2007. A Method for Automatic Membership Function Estimation Based on Fuzzy Measures. In: Foundations of Fuzzy Logic and Soft Computing, Melin, P., O. Castillo, L. Aguilar, J. Kacprzyk, W. Pedrycz (Eds.), Springer Science and Business Media, Berlin, ISBN-10: 3540729178, pp: 451-460.

Permana, K.E. and S.Z.M. Hashim, 2010. Fuzzy membership function generation using particle swarm optimization. Int. J. Open Problems Comput. Sci. Math., 3: 27-41.

Reynolds, D., 2008. Gaussian mixture models. Encyclopedia of Biometric Recognition, Springer.

Riid, A., 2002. Transparent fuzzy systems: Modeling and control. Ph.D. Thesis, Department of Computer Control, Tallinn Technical University, Estonia.

Ross, T.J., 2010. Fuzzy Logic with Engineering Applications. 3rd Edn., John Wiley and Sons, Chichester, ISBN-10: 0470748516, pp: 606.

Sala, A., T.M. Guerra and R. Babuka, 2005. Perspectives of fuzzy systems and control. Fuzzy Sets Syst., 156: 432-444. DOI: 10.1016/j.fss.2005.05.041

Sepúlveda, R., O. Montiel, O. Castillo and P. Melin, 2012. Embedding a high speed interval type-2 fuzzy controller for a real plant into an FPGA. Applied Soft Comput., 12: 988-998.

DOI: $10.1016 /$ j.asoc.2011.11.031
Turksen, I.B. and A. Celikyilmaz, 2010. Information extraction from text-dealing with imprecise data. In: Fuzzy Systems, Azar, A.T. (Ed.), InTech.

Wagner, C., 2013. Juzzy-a java based toolkit for type2 fuzzy logic. Proceedings of the IEEE Symposium on Advances in Type-2 Fuzzy Logic Systems, Apr. 16-19, IEEE Xplore Press, Singapore, pp: 45-52. DOI: $10.1109 /$ T2FZZ.2013.6613298

Wagner, C., M. Pierfitt and J. McCulloch, 2014. Juzzy online: An online toolkit for the design, implementation, execution and sharing of type- 1 and type-2 fuzzy logic systems. Proceedings of the IEEE International Conference on Fuzzy Systems, Jul. 6-11, IEEE Xplore Press, Beijing, pp: 2321-2328. DOI: 10.1109/FUZZ-IEEE.2014.6891548

Wang, L.X., 2003. The WM method completed: A flexible fuzzy system approach to data mining. IEEE Trans. Fuzzy Syst., 11: 768-782. DOI: 10.1109 /TFUZZ.2003.819839

$\mathrm{Wu}$, M.T., T.P. Hong and C.N. Lee, 2012. A continuous ant colony system framework for fuzzy data mining. Soft Comput., 16: 2071-2082. DOI: $10.1007 / \mathrm{s} 00500-012-0878-5$

$\mathrm{Wu}, \mathrm{D}$. and J.M. Mendel, 2014. Designing practical interval type-2 fuzzy logic systems made simple. Proceeding of the IEEE International Conference on Fuzzy Systems, Jul. 6-11, IEEE Xplore Press, Beijing, pp: 800-807. DOI: 10.1109/FUZZ-IEEE. 2014.6891534

Yang, C.C. and N.K. Bose, 2006. Generating fuzzy membership function with self-organizing feature map. Patt. Recognit. Lett., 27: 356-365. DOI: $10.1016 /$ j.patrec.2005.08.026

Zadeh, L.A., 1965. Fuzzy sets. Inform. Control, 8: 338-353. DOI: 10.1016/S0019-9958(65)90241-X

Zadeh, L.A., 1975. The concept of a linguistic variable and its application to approximate reasoning. Inform. Sci., 8: 199-249. DOI: $10.1016 / 0020-0255(75) 90036-5$ 